\title{
Detection of low-velocity impact-induced delaminations in composite laminates using Auto-Regressive models
}

\author{
Davide Nardi ${ }^{*, 1}$, Luca Lampani ${ }^{2}$, Michele Pasquali ${ }^{3}$, Paolo Gaudenzi ${ }^{4}$ \\ University of Rome La Sapienza, Via Eudossiana 18, Rome 00184, Italy
}

\section{A R T I C L E I N F O}

\section{Article history:}

Available online $\mathrm{xxxx}$

\section{Keywords:}

Carbon-fiber-reinforced-plastic

Low-velocity impact

Delamination

Structural health monitoring

Auto-Regressive model

\begin{abstract}
A B S T R A C T
In this paper, the detection of delaminations in carbon-fiber-reinforced-plastic (CFRP) laminate plates induced by low-velocity impacts (LVI) is investigated by means of Auto-Regressive (AR) models obtained from the time histories of the acquired responses of the composite specimens. A couple of piezoelectric patches for actuation and sensing purposes are employed. The proposed structural health monitoring (SHM) routine begins with the selection of the suitable locations of the piezoelectric transducers via the numerical analysis of the curvature mode shapes of the CFRP plates. The normalized data recorded for the undamaged plate configuration are then analyzed to obtain the most suitable AR model using five techniques based on the Akaike Information Criterion (AIC), the Akaike Final Prediction Error (FPE), the Partial Autocorrelation Function (PAF), the Root Mean Squared (RMS) of the AR residuals for different order $p$, and the Singular Value Decomposition (SVD). Linear Discriminant Analysis (LDA) is then applied on the AR model parameters to enhance the performance of the proposed delamination identification routine. Results show the effectiveness of the developed procedure when a reduced number of sensors is available.
\end{abstract}

(c) 2016 Elsevier Ltd. All rights reserved.

\section{Introduction}

Carbon-fiber-reinforced plastic (CFRP) structures have been developed and extensively implemented in the aeronautic and space industries over the last years. The major benefits of composites over other conventional materials are manifold: higher stiffness-to-weight or strength-to-weight ratio, higher resistance in harsh environments, lighter in weights Nevertheless, CFRP components can be affected by delaminations induced by low-velocity impact (LVI) inducing a breakdown of structural performances throughout their service life [1].

LVI are impacts characterized by an impact velocity smaller than $10 \mathrm{~m} / \mathrm{s}$ [2] that can produce a combination of matrix cracking, delamination and fiber breakage, among which delaminations lead to a severe stiffness and strength decrement [3]. The monitoring of

\footnotetext{
* Corresponding author.

E-mail address: davide.nardi@uniroma1.it (D. Nardi).

1 PhD Student at the Department of Mechanical and Aerospace Engineering, Italy.

2 Assistant Professor at the Department of Mechanical and Aerospace Engineering, Italy.

${ }^{3}$ Researcher Associate at the Department of Mechanical and Aerospace Engineering, Italy.

${ }^{4}$ Faculty Professor at the Department of Mechanical and Aerospace Engineering, Italy.
}

http://dx.doi.org/10.1016/j.compstruct.2016.02.005

0263-8223/@ 2016 Elsevier Ltd. All rights reserved. structural changes can be based on changes in dynamic response. In fact, any change in the physical properties, such as reduction in stiffness resulting from the onset of cracks, produces changes in the measured dynamic response of the structure [4].

The time history response of a structure can be acquired by various sensors, e.g. accelerometers, fiber optic strain gauges etc., and such measures can then be evaluated in the frequency domain using Fourier transform. Further analysis of the frequency domain data is subsequently performed to extract modal parameters of the system [5]. Modal data, however, e.g. resonance frequency, reflects the global properties of the system, whilst damage is typically a local phenomenon.

A typical structural health monitoring process requires (i) the observation of the system over time by means of sampled dynamic response measurements from sensors, (ii) the extraction of proper damage-sensitive features from such measures, and (iii) the statistical analysis of the selected features to assess the state of the system [6]. It is then apparent that the setup of an optimally-placed transducer network and the data acquisition from the structure play a crucial role. In this context, the use of piezoelectric transducers has showed to lead to prominent results. A detailed description of the mathematical modeling of piezoelectric laminated composite plates can be found in $[7,8]$.

In this article, the detection of delaminations in CFRP composite laminates subjected to LVI is accomplished. 
Table 1

Composite laminate model.

\begin{tabular}{ll}
\hline Geometrical features & \\
\hline Length $(b)[\mathrm{cm}]$ & 15 \\
Width $(a)[\mathrm{cm}]$ & 10 \\
Thickness $(t)[\mathrm{cm}]$ & 0.4 \\
Number of plies & 16 \\
Stacking sequence & {$\left[45^{\circ} / 0^{\circ} /-45^{\circ} / 90^{\circ}\right]_{2 s}$} \\
\hline
\end{tabular}

The essential parts of the proposed technique are (i) the piezo devices placement and data acquisition, (ii) the data fitting via AR model, (iii) the pattern recognition procedure, based on the separation of the measured vibration data in the reference and the comparison datasets, (iv) the classification operation and (v) the discussion of the obtained results.

This SHM routine is based on previous damage pattern recognition studies performed on composite beam [9] and plates [10] via wavelet-based approach. The novelty here reported is the use of AR parameters as damage sensitive features. Such layout represents a practical solution for an in situ monitoring procedure of the state of integrity of the structure.

\section{Piezo devices placement and data acquisition}

Three laminated composite plates are used for the impact tests. The prepreg used to manufacture the specimens is a M10.1/38\%/UD300/CHS, which consists of a thermosetting epoxide matrix with unidirectional carbon fibers. Cutting, laminating and curing are the three main phases referred to such process. Although the three composite plates are manufactured with standard geometrical dimensions and with the same materials, any physical changes are ascribable to these phases.

The active structural sensing diagnostic of the composite structure is achieved by means of a piezoelectric sensors couple that are employed for the generation of known and controlled inputs signals to excite the structure and then record its response.

The selected optimal placement of the piezo devices is the high strain region obtained via twofold differentiation of the mode shapes [11].

The geometrical parameters and stacking sequence of the laminates are reported in Table 1. The curvature of the mode shapes is obtained by implementing a finite element model of the unidirectional Carbon/Epoxy plate. The material mechanical properties adopted in the numerical model and its description can be found in [10]. The proposed work is limited to the first three resonance modes.

Due to its accurate experimental reproducibility, a free edge configuration layout is chosen for the specimen boundary conditions. Elastic bands are used to hang the laminates on a steel rigid frame.
It results that, for the first three considered modes, the $z$ component of the displacements is about ten orders of magnitude larger than $x$ and $y$ components. Therefore only the out-of-plane behavior is regarded to maximize the strain field.

A numerical interpolation through $2 \mathrm{D}$ polynomial functions of properly selected rank is carried out to extract the modal curvatures. The average surface curvature [12] is computed from the second derivatives of the approximated mode shapes with respect to $x$ and $y$ axes:

$\chi_{\text {average }}=\chi_{x}+\chi_{y}$

The average surface curvature of the considered three modes is shown in Fig.1.

The position of the piezo patches is selected so as to avoid low values of the computed average curvature ensuring, at the same time, adequate sensing and actuation conditions.

A compromise solution becomes necessary in order to assure an efficient analysis of the selected modes. In addition, the central region of the laminated plate must be avoided because in this region the impact will occur according to ASTM-D7136, together with the plates edges where impact support fixture must be placed.

The selected optimal placement of the piezo patches is shown in Fig. 2. The piezo devices are square patches of $10 \mathrm{~mm}$ length and a thickness of $0.2 \mathrm{~mm}$.

Three plates C1, C2 and C3 have been realized according to the same manufacturing process. The impact energy values considered in this study are equal to $20 \mathrm{~J}$ for $\mathrm{C} 1$ plate, $8 \mathrm{~J}$ for $\mathrm{C} 2$ plate and $12 \mathrm{~J}$ for C3 plate, which correspond to $2.7 \mathrm{~m} / \mathrm{s}, 1.7 \mathrm{~m} / \mathrm{s}$ and $2.1 \mathrm{~m} / \mathrm{s}$, respectively. For each of the above mentioned plates two configurations (before and after the impact) are considered for a total number of 6 analyzed configurations.

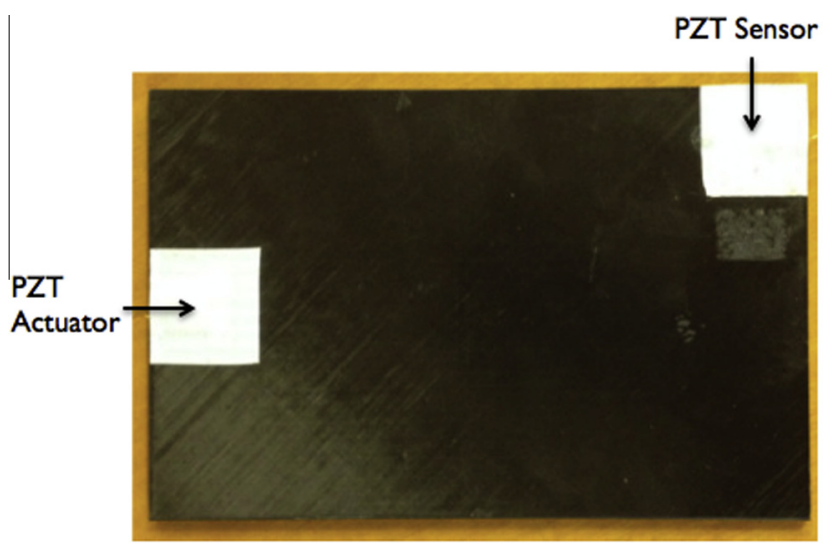

Fig. 2. The composite plate with integrated piezo patches.
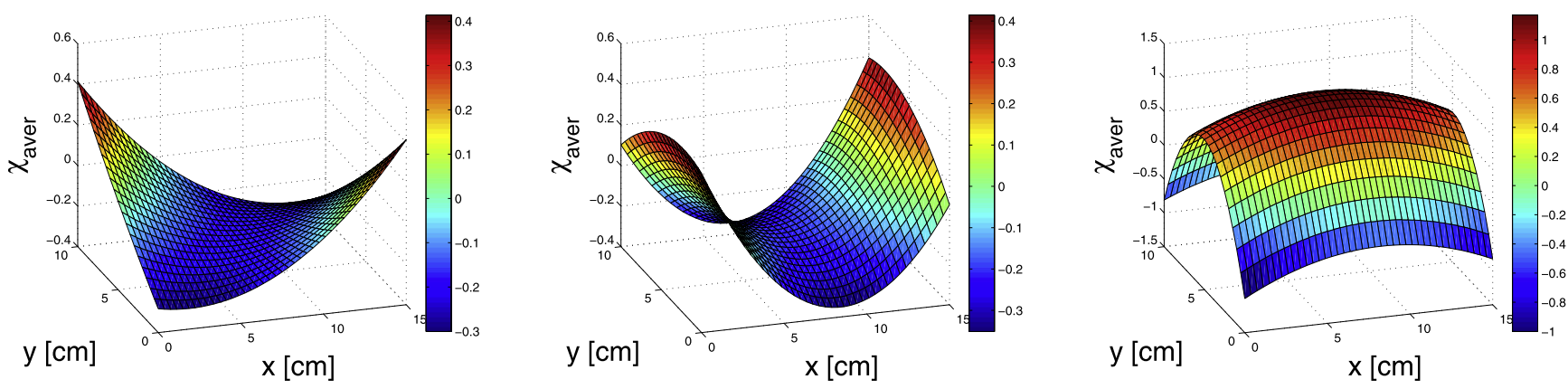

Fig. 1. Mode Shapes average curvature. From left to right: first, second and third mode shape. 
A random excitation in the range of $0-3200 \mathrm{~Hz}$ is used to excite the plates, with a frequency resolution of $1.57 \mathrm{~Hz}$. The excitation level is set to $100 \mathrm{~V}$.

Twenty time histories are acquired for each configuration in order to guarantee a proper statistical consistency. The impact tests are carried out making use of the CEAST 9340 floor standing impact system whose impactor has an hemispherical shape of $165 \mathrm{~mm}$ of diameter with a mass of $5.5 \mathrm{~kg}$ and a hardness value of $60 \mathrm{HRC}$.

\section{Data fitting via Auto-Regressive model}

A very useful model that can be adopted in the representation of time series data is the Auto-Regressive (AR) model. In such model, the current value of the process is expressed as a finite, linear ensemble of the previous values of the process and a random error [13].

The AR model is developed from sampled response time series $x_{1}, x_{2}, \ldots, x_{j}$ :

$x_{i}=\sum_{j=1}^{p} \phi_{j} x_{i-j}+\varepsilon_{i}$

where $p$ denotes the order of parameters in the model, $x_{i}$ is the measured signal at the time instant $t_{i}, \phi_{j}$ are the unknown AR parameters and $\varepsilon_{i}$ is an observable random error [16]. The unknown variables $\phi_{j}$ can be computed by means of several algorithm, e.g. the Least-squares approach and the Yule-Walker approach [17].

AR models can be exploited for SHM routine by means of two approaches: using the residual errors $\varepsilon_{i}$ or using the AR parameters $\phi_{j}$. The latter, which is employed in this work, consists of fitting an AR model to time-history data from the undamaged and damaged structural configurations and hence use them as classifier among the considered damage scenarios. The proposed damage sensitive feature is selected to overcome the limitations of other more common procedure based, for example, on the assessment of changes in the frequency response functions of the specimens [10].

The choice of the appropriate AR model order is a primary issue. A higher order model can better fit the data but can not be generalized to other data sets. Conversely, a lower model order may not properly represent the system physical dynamics [16].

Several mathematical formulation can be employed to select the AR model order. Some of them require an a priori estimate of the model parameters. Akaike's Information Criterion (AIC), Akaike's Final Prediction Error (FPE), Partial Autocorrelation Function (PAF) and Root Mean Square (RMS) have attracted much attention in the SHM literature. Other techniques do not require an a priori estimate of the model parameters. Such approaches are typically based on the Singular Value Decomposition (SVD) of the data covariance matrix [16].

\subsection{AR model order estimation}

The evaluation of the proper AR model order is performed using the time histories of the undamaged configurations of the three composite plates.

Firstly, the 20 acquired time histories of each configuration are averaged and then normalized such that

$\hat{x}=\frac{x-\mu_{x}}{\sigma_{x}}$

where $\hat{x}$ is the normalized signal and $\mu_{x}$ and $\sigma_{x}$ are the mean and standard deviation of $x$, respectively.

The results of the AR model order selection using the mentioned techniques for $\mathrm{C} 1$ plate in its undamaged configuration are shown in Fig. 3. From these plots it can be observed that while for the FPE,
RMSE and SVD functions a convergence value can be estimated around $p=45$ (see Fig. 3b, d and e, respectively), AIC function does not converge even for $p=70$ (see Fig. 3a). Also with the PAF technique the coefficients do not lie within the $95 \%$ confidence interval (see Fig. 3c). However, due to the impossibility to state a unique order for all of the estimation criterion, the results suggest that an adequate model order to represent the undamaged configuration of plate $\mathrm{C} 1$ is $p=45$.

Same results are obtained when the procedure is performed on the time histories of the undamaged configurations C2 and C3.

\subsection{AR parameters as damage sensitive feature}

All the collected time histories of the undamaged and damaged configurations of the three composite plates are normalized according to Eq. (3).

In order to consider the influence of the AR model order on the proposed damage detection procedure, four AR models, namely AR (5), $A R(15), A R(25)$, and $\operatorname{AR}(45)$, are considered.

Fig. 4a shows the values of the AR(45) model parameters for the considered six configurations using the least square technique.

It is apparent from Fig. 4 that the value of the AR parameters cannot be directly employed for classification purposes. In fact, even if some differences between the patterns can be identified, it remains difficult to discriminate the configurations easily and also to assess which AR parameters are the most sensitive ones.

In addition to the previous remark, the absolute differences between the $i$ th $\operatorname{AR}(45)$ parameters estimated for the undamaged and the damaged configuration of the $\mathrm{C} 1$ specimen are shown in Fig. 5. It can be seen that no damage-trend can be properly identified.

Similar results are obtained for C2 and C3 specimens.

To move to an effective damage pattern recognition procedure, it is then necessary to carry out statistical modeling for efficient feature classification. Such task is performed by means of the Linear Discriminant Analysis (LDA).

\section{Pattern recognition procedure}

Any pattern recognition procedure involves the classification of objects or patterns. The recognition issues refer the identification and the classification of the individual characters and to label the features according to category, e.g. regular or irregular [14].

The first step of a pattern recognition network design is to study the distributions of samples belonging to the different configurations. This phase is generally defined as learning or training and the used samples as training set. The learning phase include also the analyses of effective features, i.e. the feature extraction, which is generally defined as the process of mapping the original samples in a more effective feature subspace [15].

The Linear Discriminant Analysis (LDA) approach consists in finding a linear transformation to project the samples of each class (or configuration), defined in terms of AR parameters, into a new sub-space where the dispersion of data belonging to the same class is the smallest possible, ensuring at the same time the maximum dispersion among data of different classes. LDA originates from the Karhurnen-Loeve Expansion (KLE), also known as Proper Orthogonal Decomposition (POD).

We can define three different scatter matrices [9]: the classscatter matrix $\mathbf{S}_{\mathbf{c}}$, the within-class scatter matrix $\mathbf{S}_{\mathbf{w}}$, and the between-class scatter matrix $\mathbf{S}_{\mathbf{b}}$.

The class-scatter matrix $\mathbf{S}_{\mathbf{c}}$ expresses the scattering of the samples belonging to the same class (configuration). The within-class scatter matrix $\mathbf{S}_{\mathbf{w}}$ is the $p$-class probability weighted sum of previously defined class scatter matrix and the between-class scatter 
(a)

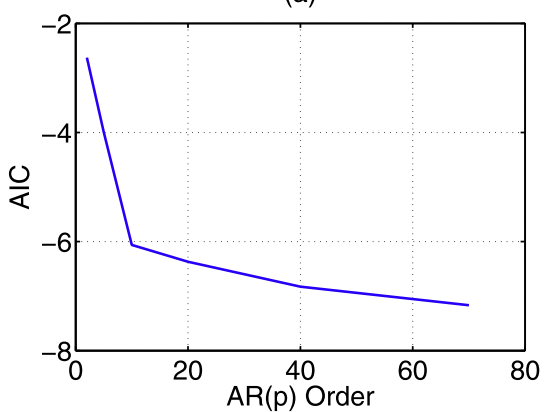

(b)

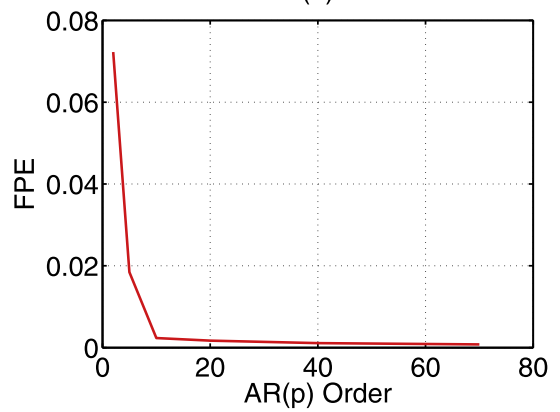

(c)

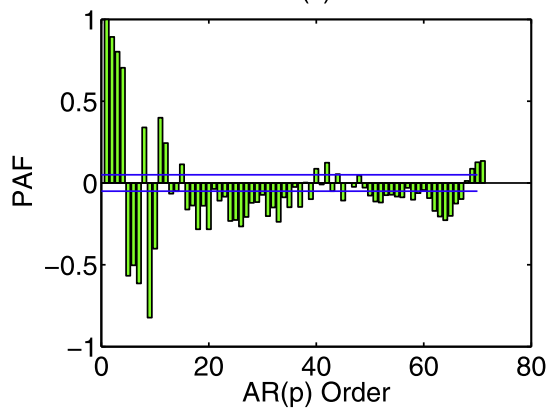

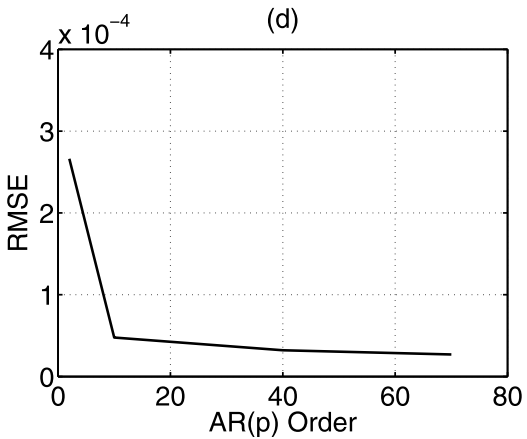

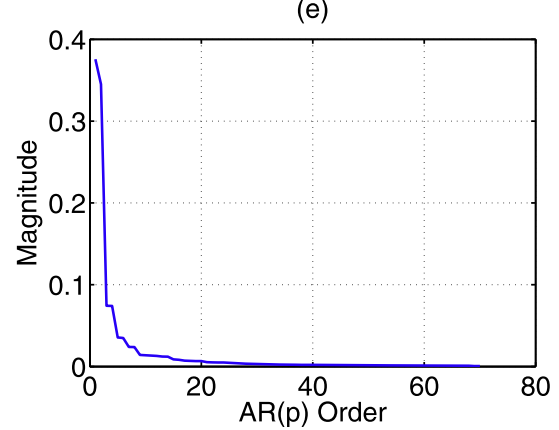

Fig. 3. AR model order estimation for C1 undamaged: AIC (a), FPE (b), PAF (c), RMSE (d) and SVD (e) criteria.

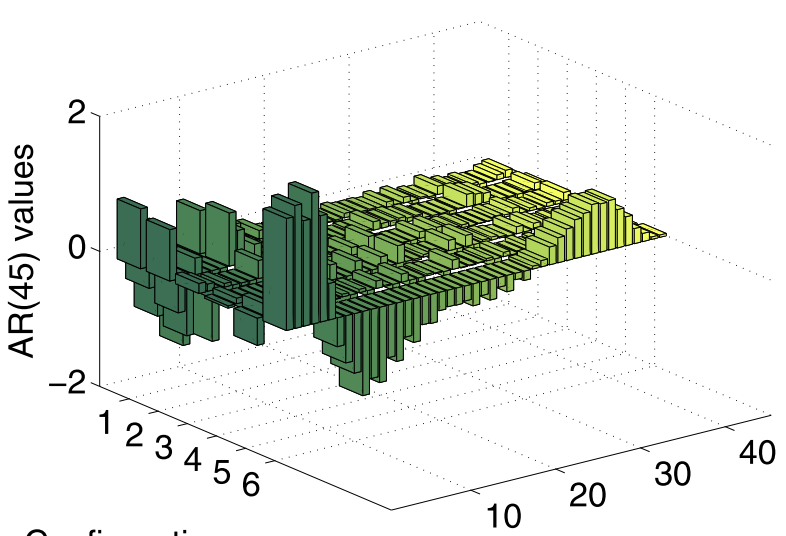

Configurations

$\mathrm{AR}(45)$ parameters

Fig. 4. AR parameters distribution over the considered configurations. Model order value $p=45$.

matrix $\mathbf{S}_{\mathbf{b}}$ expresses the scattering of mean vectors of classes with respect to the mean vector of the mean vectors [10]. From the product between the inverse matrix of $\mathbf{S}_{w}$ and the matrix $\mathbf{S}_{b}$ it is possible to obtain a $m \times m$ matrix (where $m$ is the number of the adopted AR parameters) named $\mathbf{V}$ representing the ratio of the scattering between different classes and the scattering within the same class. An effective classification procedure is achieved if the scattering of samples collected between different classes is large and, simultaneously, such scattering is small within every class.

The variables which maximize the discriminant capability of the classifier are the eigenvalues/eigenvectors of the matrix $\mathbf{V}$ [15].

The eigenvector matrix ensures a projection of the starting state variables space (the AR parameters of each class) in a sub-space in which separability among classes is the largest possible. Moreover, since every eigenvalue (with the corresponding eigenvector) is an

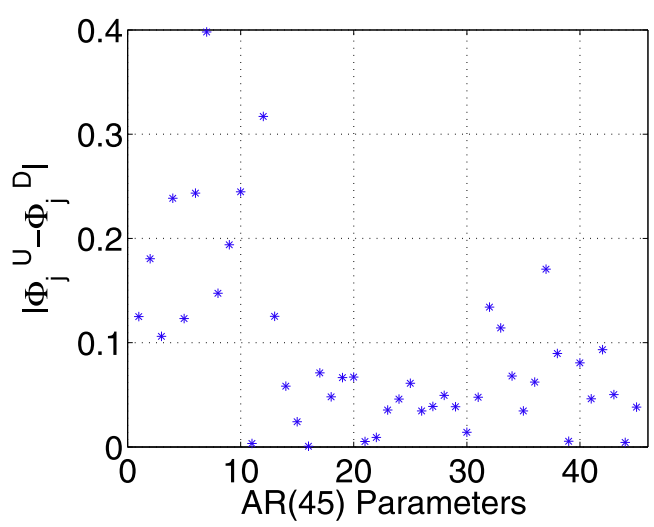

Fig. 5. Absolute differences between the undamaged $\Phi_{j}^{U}$ and damaged $\Phi_{j}^{D}$ parameters of the $\mathrm{AR}(45)$ models of plate $\mathrm{C} 1$.

index of the energy content of the whole process which takes into account all the six configurations, a number of eigenvectors $q$ corresponding to a selected energy level can be selected. The space of reduced variables is then obtained from the eigenvectors matrix selecting the rows and the columns of the first $q$ eigenvectors. For example, it can be observed from Fig. 6 (which refers to the case of AR model with $p=45$ ) that if the first 3 eigenvalues are considered, the energy content of the process is equal to $98.98 \%$ of the energy of the whole process.

\section{Classification operation}

Classification operations can be summarized in three main steps: (i) implementation of a reference dataset in the sub-space, taking the mean matrices of the AR parameters obtained from the first block of ten acquired time histories of each configuration; 


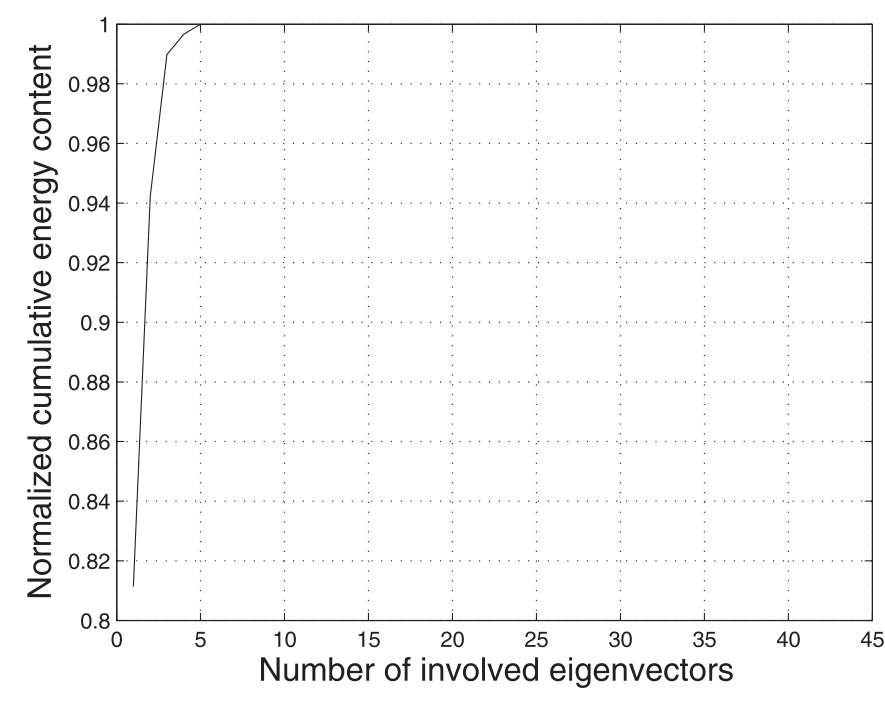

Fig. 6. Plot of the normalized cumulative energy content of the eigenvalues in increasing order.

Table 2

Results of the identification of the specimen in its (U) undamaged and (D) damaged configuration.

\begin{tabular}{lllll}
\hline \multicolumn{2}{l}{ Correct identification $(\%)$} & & & \\
\hline $\mathrm{AR}(\mathrm{P})$ & 5 & 15 & 25 & 45 \\
\hline$C 1_{U}$ & 80 & 100 & 100 & 100 \\
$C 1_{D}$ & 100 & 80 & 100 & 100 \\
$C 2_{U}$ & 100 & 80 & 100 & 70 \\
$C 2_{D}$ & 80 & 100 & 100 & 100 \\
$C 3_{U}$ & 100 & 100 & 100 & 100 \\
$C 3_{D}$ & 100 & 100 & 100 & 100 \\
\hline
\end{tabular}

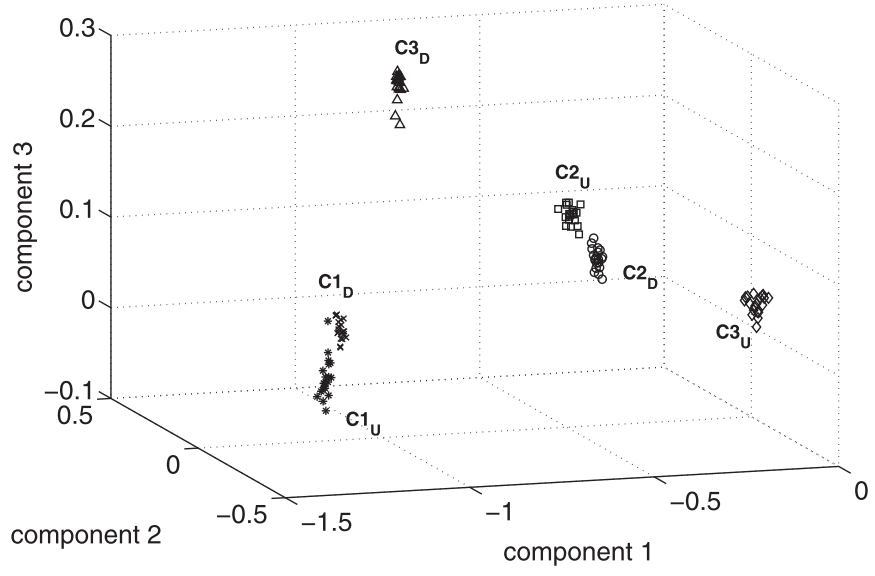

Fig. 7. Reduced dataset in the reduced state space: $C 1_{U}$ (asterisk), $C 1_{D}$ (cross), $C 2_{U}$ (square), $C 2_{D}$ (circle), $C 3_{U}$ (diamond), $C 3_{D}$ (triangle). AR model order $p=25$.

(ii) definition of a comparison dataset formed by the AR parameters obtained from the second block of ten time history of each configuration and mapped to the eigenvector sub-space and which is meant to be used as a case study; (iii) estimation of the normalized Euclidean distance between the comparison database and the reference database, namely:

$D_{k}=\frac{\left\|d_{k}\right\|}{\max \left(\left\|d_{k}\right\|\right)}=\frac{\sqrt{\sum_{i}^{P}\left(a_{i, k}-g_{i}\right)^{2}}}{\max \left(\left\|d_{k}\right\|\right)}$ where $a$ is the unknown reduced configuration vector, $g$ is the vector of pattern, and $d$ is the distance vector related to the $k$ th vector of the pattern.

According to the minimum Euclidean distance expressed in (4), the algorithm is meant to match all the distances with the corresponding configuration.

An initialization phase characterized by the identification of known damaged scenario is necessary to train the proposed algorithm.

\section{Results and conclusions}

In order to test the effectiveness of the proposed algorithm an increasing order for the AR model was considered, in particular $\mathbf{P}=[5,15,25,45]$ is investigated. The number of eigenvectors is set equal to 3 which represents an energy content of the whole process between $99 \%$ and $100 \%$ for each considered AR model order.

The classification results are expressed in terms of percentage of correct identification, as shown in Table 2.

It can be noticed that when $p=5$ and $p=15100 \%$ of the correct identification is obtained for four configurations only. For $p=25$ the correct identification is equal to $100 \%$ for all configurations, while for $p=45100 \%$ of the correct identification is obtained for five out of six configurations (see Table 2). The last result can be addressed to the phenomenon called overfitting. In fact, the performance of the considered AR model order selection criteria are optimal only if the model of the selected order is the most accurate model in the considered set of estimated models, and this is not necessarily the true model order. [18]. Therefore, for the considered damage detection procedure, the optimal AR model order is 25 . However, in all of the considered cases, the worst identification percentage is not lower than $70 \%$.

Fig. 7 shows the distribution of the AR model parameters in the reduced three-dimension space for $p=25$ : the groups of samples of the same configuration occupy a clear location in the 3D reduced eigenvectors space.

The proposed SHM routine showed high percentages of success in the identification of delamination-induced damage in composite plates using a reduced number of sensors.

The procedure, based on signal analysis of the vibration data, is characterized by a learning phase of the proposed damage detection algorithm with a reference dataset of the acquired normalized time histories and by a matching with a comparison dataset of another group of acquired normalized time histories of the considered configurations.

Such approach, based on signal analysis of the acquired vibration data, appears to be particularly suitable for the development of an automated health monitoring system of high performance materials.

\section{References}

[1] Lin M, Chang F. The manufacture of composite structures with a built-in network of piezoceramics. Compos Sci Technol 2002;62:919-39.

[2] Sjoblom P, Hartness J, Cordell T. On low-velocity impact testing of composite materials. J Compos Mater 1988;22:30-52.

[3] Perez M, Gil L, Oller S. Impact damage identification in composite laminates using vibration testing. Compos Struct 2014;108:267-76.

[4] Sohn H, Farrar C, Hunter N, Worden K. Structural health monitoring using statistical pattern recognition techniques. Trans ASME 2001;123:706-11.

[5] Carden E, Fanning P. Vibration based condition monitoring:a review. Struct Health Monit 2004;3:355-77.

[6] Sohn H, Farrar C. Damage diagnosis using time series analysis of vibration signals. Smart Mater Struct 2001;10:446-51.

[7] Gaudenzi P. Smart structures. Physical behaviour, mathematical modeling and applications. Wiley; 2009.

[8] Reddy J. On laminated composite plates with integrated sensors and actuators. Eng Struct 1999;21:568-93. 
[9] Facchini G, Bernardini L, Atek S, Gaudenzi P. Use of the wavelet packet transform for pattern recognition in a structural health monitoring application. J Intell Mater Syst Struct 2015;26:1513-29.

[10] Gaudenzi P, Nardi D, Chiappetta I, Atek S, Lampani L, Pasquali M, Sarasini F, Tirilló J, Valente T. Sparse sensing detection of impact-induced delaminations in composite laminates. Compos Struct 2015;133:1209-19.

[11] Crawley E, De Luis J. Use of the piezoelectric actuators as elements of intelligent structures. AIAA J 1987;25:1373-85.

[12] Timoshenko S, Woinowsky-Krieger S. Theory of plates and shells. McGrawHill; 1987.

[13] Box GE, Jenkins GM, Reinsel GC. Time series analysis: forecasting and control. Prentice-Hall; 1994.
[14] Jain AK, Dubes RC. Algorithms for clustering data. Prentice-Hall; 1988.

[15] Fukunaga K. Introduction to statistical pattern recognition. Academic Press Professional; 1990.

[16] Figueiredo E, Figueiras J, Park G, Farrar C. Influence of the autoregressive model order on damage detection. Comput-Aided Civil Infrastruct Eng 2011;26:225-38.

[17] Marple S. Digital spectral analysis with applications. Prentice-Hall; 1987.

[18] De Waele S, Broersen PMT. Order selection for vector autoregressive models. IEEE Trans Signal Process 2003;51(427):433. 